\title{
O problema da sobreutilização de recursos e o projeto Choosing Wisely Portugal - Escolhas Criteriosas em Saúde
}

\author{
The problem of overutilization of resources and the Choosing \\ Wisely Portugal program - Judicious Choices in Health
}

Miguel Bigotte Vieira (https://orcid.org/0000-0003-0528-2716) ${ }^{1,2}$

António Vaz-Carneiro (https://orcid.org/0000-0002-2783-0544) ${ }^{2}$

${ }^{1}$ Serviço de Nefrologia, Hospital Curry Cabral, Centro Hospitalar Universitário de Lisboa Central. R. da Beneficência 8. 1050-099 Lisboa Portugal.

mbigottevieira@gmail.com ${ }^{2}$ Instituto de Saúde Baseada na Evidência, Universidade de Lisboa. Lisboa Portugal.

\begin{abstract}
The variation of clinical practice is a well-described phenomenon in which patients with similar diagnoses, prognoses and demographic status receive different levels of care depending on when, where and by whom they are treated, despite agreed and documented evidence of best practice. The overutilization of health resources is also known to have a negative risk-benefit ratio. Unfortunately, both phenomena occur in several countries, especially in developed countries. In the light of this, the American Board of Internal Medicine launched the Choosing Wisely campaign, which has subsequently been implemented in several countries, including Portugal.

Key words Variation in clinical practice, Choosing Wisely, Judicious Choices in Health, Informed Decision, Shared Decision
\end{abstract}

Resumo A variação da prática clínica é um fenómeno que se encontra bem descrito e que se caracteriza por uma prática clínica utilizando testes diagnósticos e esquemas terapêuticos diversos, em doentes semelhantes na patologia que apresentam e no seu risco basal. A sobreutilização de recursos em saúde caracteriza-se por apresentar um benefício-risco negativo. Infelizmente ambos os fenómenos se verificam em diversos países, em particular países desenvolvidos. Neste contexto, o American Board of Internal Medicine lançou a campanha Choosing Wisely, a qual foi, entretanto, implementada em diversos países incluindo Portugal. Palavras-chave Variação da prática clínica, Choosing Wisely, Escolhas Criteriosas em Saúde, Decisão Informada, Decisão Partilhada 


\section{Variação da prática clínica e a sobreutilização de recursos}

Nos anos 1980 foi detectado pelos serviços de saúde ingleses que doentes com o mesmo tipo de tumor da mama (em termos de padrão histológico, agressividade e estadiamento) eram tratadas de maneira diversa, dependendo do local onde viviam: nuns locais eram predominantemente operadas, noutros faziam radioterapia, noutros ainda faziam quimioterapia ou hormonoterapia.

Este fenómeno designa-se por variação da prática clínica, que se define como uma prática utilizando testes diagnósticos e esquemas terapêuticos diversos, em doentes semelhantes na patologia que apresentam e no seu risco basal. Isto é: as abordagens práticas não são explicáveis pelas diferenças clínicas individuais dos doentes (por exemplo nos casos em que não existe uma solução clara e inequívoca, podendo haver alternativas igualmente válidas), mas sim pelas diferenças nos padrões de prática dos médicos ${ }^{1}$.

Em Portugal as taxas de variação são semelhantes às de outros Países europeus, abrangendo variadas áreas clínicas. Por exemplo, na Figura 1 e no Quadro 1 pode ver-se a variação da utilização da angioplastia coronária percutânea (PTCA) nas diversas regiões do País.

Apesar de uma prevalência de doença coronária relativamente constante a nível nacional, verifica-se uma variação significativa dos procedimentos com um aumento consistente da sua frequência de utilização² .

Quem melhor tem estudado a variação da prática clínica é o grupo americano de Dartmouth, que publica o conhecido Dartmouth Atlas of Health $\mathrm{Care}^{3}$. As análises feitas por este grupo permitiram classificar os cuidados em três tipos:

1 - Cuidados efectivos (effective care), que são os serviços que, baseados na melhor evidência científica, são melhores que as alternativas disponíveis, já que os benefícios do tratamento ultrapassam largamente os riscos (em termos de efeitos secundários ou complicações clínicas). Em princípio, todos os doentes elegíveis devem ter este tipo de cuidados, a não ser que haja alguma contra-indicação individual específica.

2 - Cuidados eletivos (preference sensitive), que são cuidados com mais do que uma opção clínica, induzindo níveis de qualidade de vida muito diferentes, mas com resultados semelhantes no seu benefício. Neste caso, a selecção dos esque-

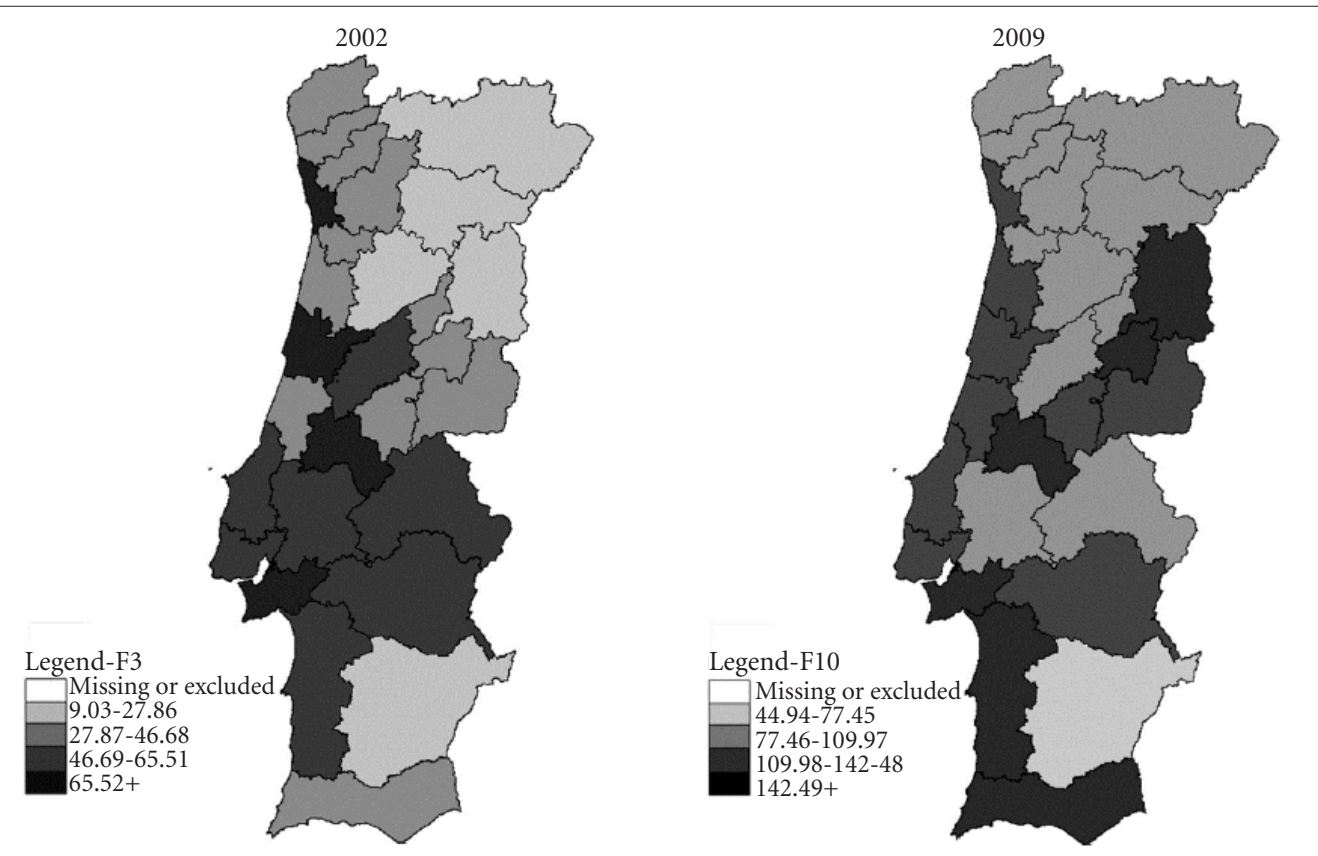

Figura 1. Mapas da taxa padronizada de angioplastia coronária percutânea por 100.000 habitantes, por região geográfica. Portugal, 2002 e 2009. 
Quadro 1. Taxa padronizada de angioplastia coronária percutânea por 100.000 habitantes. Portugal, 2002-2009.

\begin{tabular}{|l|c|c|c|c|c|c|c|c|}
\hline & $\mathbf{2 0 0 2}$ & $\mathbf{2 0 0 3}$ & $\mathbf{2 0 0 4}$ & $\mathbf{2 0 0 5}$ & $\mathbf{2 0 0 6}$ & $\mathbf{2 0 0 7}$ & $\mathbf{2 0 0 8}$ & $\mathbf{2 0 0 9}$ \\
\hline Média não ponderada & 43 & 55 & 68 & 79 & 89 & 108 & 121 & 117 \\
\hline Q10 & 20 & 26 & 31 & 39 & 50 & 69 & 86 & 83 \\
\hline Q90 & 70 & 86 & 113 & 118 & 138 & 153 & 166 & 154 \\
\hline Coeficiente de variação & 0,46 & 0,48 & 0,48 & 0,42 & 0,39 & 0,29 & 0,33 & 0,27 \\
\hline
\end{tabular}

Fonte: Organização para a Cooperação e Desenvolvimento Económico².

mas disponíveis deveria idealmente ser feita acima de tudo pelos doentes, após terem sido detalhadamente informados das consequências de cada opção. Ora, porque estes delegam a maior parte das vezes essas decisões nos médicos, é a opinião destes que acaba por prevalecer na decisão final: cirurgia versus terapêutica médica, intervenção simples versus complexa, maior versus menor intensidade de tratamento, etc. $\mathrm{O}$ caso típico é o doente oncológico, que se for visto por um oncologista terá tendência a ser tratado com fármacos, se for assistido por um cirurgião poderá ser-lhe proposta uma solução cirúrgica e que se for ao radiologista fará muito provavelmente radioterapia.

3 - Cuidados sensiveis à oferta (supply sensitive). Esta categoria clínica refere-se acima de tudo à frequência com que são oferecidos aos doentes os cuidados disponíveis, em que quanto maior a oferta, maior o consumo. Estes serviços incluem consultas médicas, exames diagnósticos, internamentos hospitalares, etc. e esta prática verifica-se maioritariamente em contexto de doença crónica, em que existem múltiplas opções possíveis. Como já afirmámos anteriormente, "a selecção dos cuidados sensíveis à oferta existe quando precisamente há muita oferta de serviços - especialistas, consultas médicas, tecnologias de imagem, camas em unidades de cuidados intensivos, etc. - e, portanto, elevado consumo dos mesmos. As decisões médicas neste caso são poderosamente influenciadas pela disponibilidade das tecnologias e das capacidades existentes nas unidades de saúde, estando subjacente por trás a ideia (errada) que quanto maior é a intensidade de cuidados, melhores estes serão (Figuras 2 e 3). A ausência de consenso de quais serão as melhores práticas para todas as possíveis situações clínicas ajuda a este tipo de consumerismo médico, raramente baseado na evidência e muito prevalente em certos subsistemas de saúde. Este tipo de cuidados é claramente muito mais dispendioso e curiosamente os doentes - apesar de eles próprios esperarem maior intensidade de cuidados - acabam por classificar as suas experiências como piores, passando mais dias internados, a serem vistos por muito mais médicos, com sistemas mais desorganizados e maiores taxas de resultados negativos"4.

As Figuras 2 e 3 - do grupo de Dartmouth - representam, respectivamente, a relação entre

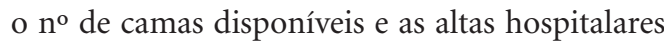
(em que o no de admissões hospitalares está directamente relacionado com o $\mathrm{n}^{\circ}$ de camas disponíveis) e a relação entre a oferta de cuidados cardiológicos e o $\mathrm{n}^{\mathrm{o}}$ de consultas efectuadas (em que quanto mais cardiologistas há disponíveis maior é o no de consultas efectuadas) (reproduzidas com permissão).

Na prática, e segundo análises detalhadas de padrões de actuação, parece ser possível afirmar que existe uma subutilização dos cuidados efectivos, uma má utilização dos cuidados electivos e uma sobreutilização dos cuidados sensíveis à oferta (Dartmouth).

Neste último caso é que está incluído o conceito de sobreutilização de recursos em saúde, entendido como uma prática que, quando implementada, terá como resultados um balanço benefício-risco negativo: em linguagem coloquial, os serviços farão mais mal que bem ${ }^{5}$.

Para medir directamente este uso excessivo de recursos, é obviamente necessária uma definição do que se considera ser a prática correcta, com base em evidências científicas que consideram o equilíbrio entre benefícios e danos para o indivíduo ou para uma população específica ${ }^{6}$.

Esta quantificação é frequentemente problemática, por três ordens de razões: 1) porque os dados sobre os benefícios são muitas vezes incompletos, 2) para muitos serviços os danos são mal documentados e 3) o limiar entre cuidados adequados e inadequados pode variar entre doentes individuais ou em grupos.

Embora os estudos sobre sobreutilização que também é designada como sobreutilização, sobremedicalização ou cuidados de baixo valor tenham sido realizados na sua maioria em países 


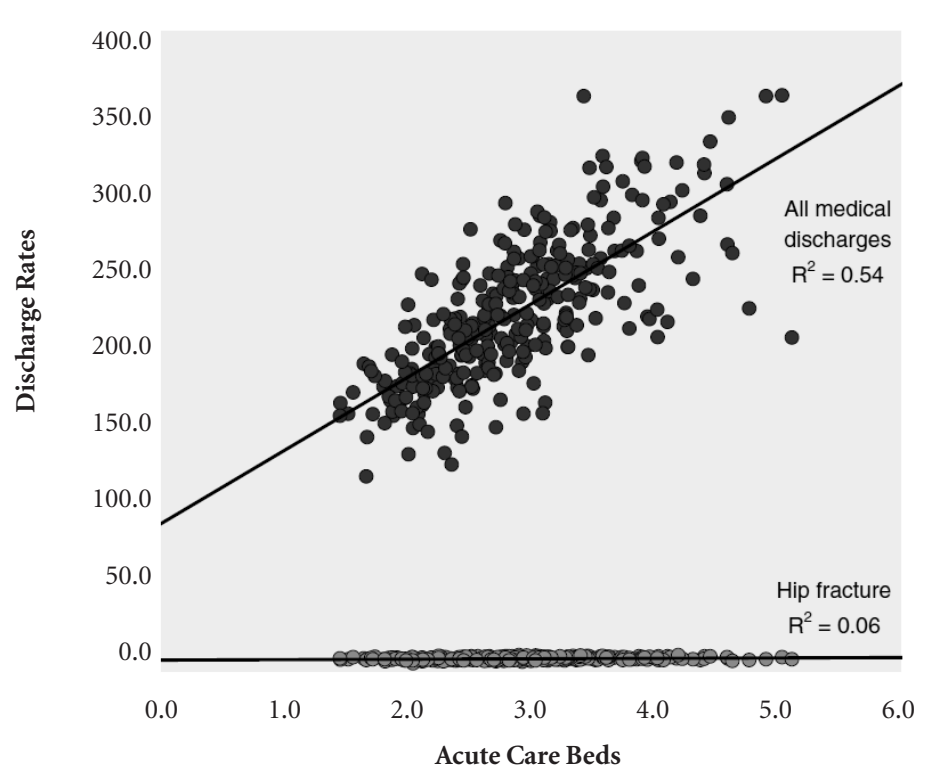

Figura 2. Relação entre o número de camas disponíveis e as altas hospitalares em 306 hospitais americanos (1996).

Fonte: Dartmouth Atlas Project ${ }^{3}$.

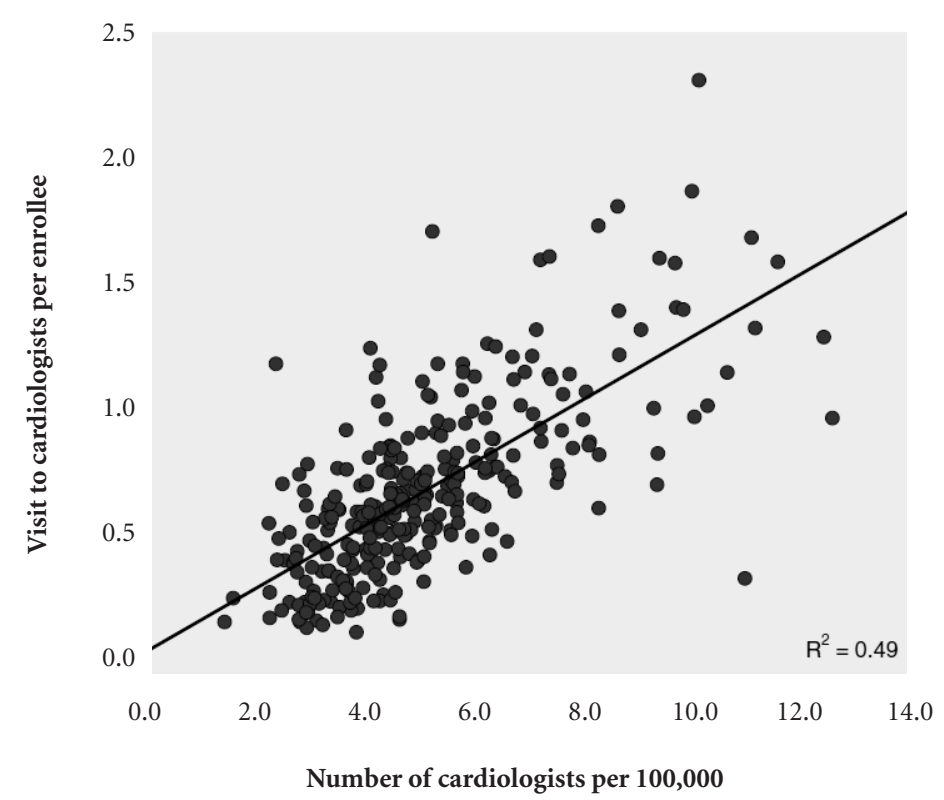

Figura 3. Relação entre o número de cardiologistas e o número de consultas cardiológicas.

Fonte: Dartmouth Atlas Project ${ }^{3}$. 
economicamente desenvolvidos, existe evidência que parece confirmar a ideia que este é um fenómeno global, independente da riqueza ou do PIB dos países. Internacionalmente, a sobreutilização parece situar-se entre $10 \%$ e $30 \%$, dependendo das fontes utilizadas ${ }^{1}$.

Uma das áreas mais analisadas é a dos rastreios de massas, cujos benefícios e riscos têm vindo a ser cuidadosamente analisados, com recomendações subjacentes de abandono destas práticas de detecção de doença assintomática numa percentagem significativa de casos ${ }^{7}$.

Os casos típicos são os rastreios dos cânceres da mama e próstata, mas outros tumores estão sob o mesmo escrutínio ${ }^{8,9}$.

No caso dos benefícios/danos do rastreio do cancro da mama, o Quadro 2 descreve o seu potencial impacto numa coorte de 10.000 mulheres rastreadas anualmente durante 10 anos $^{10}$.

Para contrariar este estado de coisas, foi lançada pelo American Board of Internal Medicine a campanha Choosing Wisely, neste momento presente em vários Países (incluindo Portugal).

\section{O Projecto Choosing Wisely Portugal - Escolhas Criteriosas em Saúde}

O programa Choosing Wisely é um programa de educação para a saúde fundado em 2012 pelo American Board of Internal Medicine ${ }^{11}$. O programa tem como objetivo promover escolhas em Saúde baseadas na melhor evidência científica e reduzir o número de intervenções desnecessárias, sem eficácia comprovada e/ou com uma relação risco-benefício desfavorável. Este programa foi posteriormente adaptado e implementado em diversos países.

Quadro 2. Estimativas de benefício/dano de rastreio anual com mamografia em 10.000 mulheres durante 10 anos.

\begin{tabular}{|l|r|r|r|}
\hline & \multicolumn{3}{|c|}{ Grupo etário } \\
\hline Idade (anos) & 40 & 50 & 60 \\
\hline $\begin{array}{l}\text { Diagnóstico de cancro da } \\
\text { mama invasivo }\end{array}$ & 147 & 231 & 345 \\
\hline Mortes por cancro da mama & 32 & 62 & 88 \\
\hline $\begin{array}{l}\text { Mortes evitadas por cancro } \\
\text { da mama }\end{array}$ & 3 & 10 & 43 \\
\hline$\geq 1$ falso positivo & 6.130 & 6.130 & 4.970 \\
\hline$\geq 1$ biópsia desnecessária & 700 & 940 & 980 \\
\hline Sobrediagnóstico (n) & 28 & 44 & 66 \\
\hline $\begin{array}{l}\text { Fonte: Adaptado de Keating e Pace }{ }^{10} . \\
\end{array}$
\end{tabular}

A nível internacional o programa tem cinco princípios fundamentais: deve ser liderado por médicos, centrado no doente, baseado na evidência científica disponível à data da elaboração de cada recomendação, multidisciplinar e transparente $^{12}$. Em Portugal o programa intitula-se Choosing Wisely Portugal - Escolhas Criteriosas em Saúde e a sua implementação foi iniciada em outubro de 2018.

O problema da sobreutilização de recursos em saúde encontra-se bem identificado em Portugal. Num estudo realizado em 2013 que inquiriu 1.000 portugueses, cerca de $99 \%$ considerava que deveria realizar análises de rotina anualmente e 70\% que deveria realizar radiografias de tórax a cada 15 meses $^{13}$. Um estudo posterior do mesmo grupo avaliou a importância atribuída pelos doentes a várias medidas preventivas e concluiu que os doentes tendem a sobrestimar a importância de medidas preventivas, a atribuir maior importância à realização de exames do que a alterações de estilo de vida, a não identificar quais os exames cuja realização se baseia em evidência e a desconhecer o conceito de individualização de risco ${ }^{14}$. Tendo por base os resultados deste estudo compreende-se que seja frequente a solicitação deste tipo de intervenções a profissionais de saúde em Portugal. Neste contexto, a implementação deste programa em Portugal pretendeu promover escolhas criteriosas em saúde e facilitar a comunicação entre profissionais de saúde e doentes.

A implementação prévia deste programa em diversos países permitiu-nos aprender com a experiência entretanto adquirida ${ }^{12}$. Considerámos que o programa devia ser promovido por uma entidade cientificamente competente, idónea e independente das envolvidas no financiamento dos serviços de saúde - em Portugal, a Ordem dos Médicos portuguesa.

Por este motivo, as recomendações do programa Choosing Wisely Portugal - Escolhas Criteriosas em Saúde são elaboradas por médicos peritos e emitidas pelos Colégios da Especialidade da Ordem dos Médicos, incidindo preferencialmente sobre procedimentos frequentes na prática clínica. As recomendações emitidas são produzidas de novo ou têm por base recomendações de programas Choosing Wisely internacionais, que são traduzidas e adaptadas à realidade portuguesa. De modo a incentivar a colaboração entre especialidades médicas existem recomendações elaboradas em conjunto por diferentes Colégios de Especialidade e recomendações emitidas por um único Colégio da Especialidade podem também ser subscritas por outros Colégios. 
As recomendações baseiam-se na melhor evidência científica disponível à data da sua elaboração, são escritas em linguagem compreensível por leigos, são identificadas com os logótipos do programa e da Ordem dos Médicos portuguesa e estão disponíveis para consulta e download nas redes sociais e na página do programa, o qual se encontra integrado no site da Ordem dos Médi$\cos$ portuguesa ${ }^{15,16}$. Foram estabelecidas parcerias com outras entidades na área da saúde de modo a aumentar a divulgação do programa, incluindo sociedades científicas e associações de doentes. Foi ainda estabelecida parceria com meios de comunicação social de modo a que os conteúdos do programa estejam também acessíveis em sites frequentemente consultados pelo público em geral. Foram ainda solicitadas sugestões a todos os parceiros no sentido de melhorar continuamente o programa.

De modo a avaliar a percepção dos médicos portugueses sobre o programa Choosing Wisely Portugal - Escolhas Criteriosas em Saúde foi realizado em abril de 2019 um inquérito electrónico a todos os médicos inscritos na Ordem dos Médicos portuguesa. Este inquérito teve como objetivo avaliar a opinião e atitude dos médicos portugueses relativamente à sobreutilização de recursos em saúde e ao próprio programa em Portugal ${ }^{17}$. Foram obtidas 2.684 respostas, tendo a maioria dos médicos $(95 \%)$ considerado que a realização de exames complementares de diagnóstico e procedimentos desnecessários era um problema. Cerca de $50 \%$ referiu que na sua prática clínica lhe era solicitada a prescrição de exames desnecessários pelo menos uma vez por semana. Por outro lado, apenas $65 \%$ referia que quase sempre, ou frequentemente, os doentes seguiam o seu conselho e evitavam a realização de exames complementares de diagnóstico desnecessários. Neste estudo, os principais motivos que levaram os médicos a prescrever um exame ou procedimento desnecessário em Portugal foram a necessidade de mais informação para fundamentar a sua atitude clínica $(62 \%)$, o receio de ser alvo de processos por má prática médica (24\%) e a insistência do próprio doente $(21 \%)$. Consideramos que estes dados são paradigmáticos da extensão do problema de sobreutilização de recursos em saúde e da dificuldade na sua resolução. De salientar ainda que no mesmo estudo o aumento dos tempos de consulta era referido como uma medida potencialmente eficaz para resolver este problema por $92 \%$ dos médicos. Neste contexto, tem sido realizado um esforço pela Ordem dos Médicos portuguesa no sentido de definir um tempo mínimo de realização de consulta médica, adaptado à situação particular de cada especialidade médica ${ }^{18}$.

No final de outubro de 2019 já tinham aderido ao programa 28 Colégios da Especialidade da Ordem dos Médicos portuguesa e sido emitidas 139 recomendações. Vinte e uma sociedades científicas já tinham manifestado o seu apoio e disponibilidade em colaborar neste programa e 18 associações de doentes e entidades parceiras apoiavam e colaboravam na sua divulgação. Encontra-se prevista para 2020 a repetição do inquérito junto dos médicos e a comparação dos resultados com os obtidos em 2019. Esta avaliação será importante para reavaliar a opinião dos profissionais de saúde sobre o programa Choosing Wisely Portugal e avaliar a eficácia da divulgação do programa junto dos mesmos. O programa Choosing Wisely Portugal recebeu, entretanto, financiamento de bolsas públicas e privadas de incentivo à educação para a saúde. Nos próximos meses prosseguiremos com a divulgação do programa a nível nacional junto de profissionais de saúde e junto da população em geral bem como com a inclusão de novas recomendações e de novas especialidades médicas no programa.

Neste contexto, consideramos que a introdução e adaptação do programa Choosing Wisely a outros países poderá alertar para a sobreutilização dos recursos em saúde e facilitar aos profissionais de saúde ferramentas de educação para a saúde que lhes permitam dialogar com o doente, de modo simples e eficiente. É nossa convicção que este programa poderá contribuir para a redução do número de intervenções diagnósticas e terapêuticas desnecessárias, sem eficácia comprovada e/ou com uma relação risco-benefício desfavorável. 


\section{Colaboradores}

Ambos os autores contribuíram significativamente para a escrita e revisão do presente artigo.

\section{Referências}

1. Morgan DJ, Brownlee S, Leppin AL, Kressin N, Dhruva SS, Levin L, Landon BE, Zezza MA, Schmidt H, Saini V, Elshaug AG. Setting a research agenda for medical overuse. BMJ 2015; 351:h4534.

2. Organisation for Economic Co-operation and Development (OECD). Geographic Variations in Health Care: What Do We Know and What Can Be Done to Improve Health System Performance? OECD Health Policy Studies, OECD Publishing; 2014.

3. Dartmouth Atlas of Health Care [Internet]. [acessado 2020 jan 6]. Disponível em: https://www.dartmouthatlas.org/.

4. Carneiro AV. Mitos e Crenças na Saúde. Lisboa: Livros Horizonte; 2019.

5. Saini V, Brownlee S, Elshaug AG, Glasziou P, Heath I. Addressing overuse and underuse around the world. Lancet 2017; 390(10090):105-107.

6. Brownlee S, Chalkidou K, Doust J, Elshaug AG, Glasziou P, Heath I, Nagpal S, Saini V, Srivastava D, Chalmers K, Korenstein D. Evidence for overuse of medical services around the world. Lancet 2017; 390(10090):156-168

7. Welch HG, Brawley OW. Scrutiny-Dependent Cancer and Self-fulfilling Risk Factors. Ann Intern Med 2018; 168(2):143-144.

8. Independent UK Panel on Breast Cancer Screening. The benefits and harms of breast cancer screening: an independent review. Lancet 2012; 380(9855):17781786.

9. Fenton JJ, Weyrich MS, Durbin S, Liu Y, Bang H, Melnikow J. Prostate-Specific Antigen-Based Screening for Prostate Cancer: Evidence Report and Systematic Review for the US Preventive Services Task Force. JAMA 2018; 319(18):1914-1931.

10. Keating NL, Pace LE. Breast Cancer Screening in 2018: Time for Shared Decision Making. JAMA 2018; 319(17):1814-1815.

11. Casarett D. The Science of Choosing Wisely--Overcoming the Therapeutic Illusion. N Engl J Med 2016; 374(13):1203-1205.

12. Levinson W, Kallewaard M, Bhatia RS, Wolfson D, Shortt S, Kerr EA, Choosing Wisely International Working Group. "Choosing Wisely": a growing international campaign. BMJ Qual Saf 2015; 24(2):167174.

13. Martins C, Azevedo LF. Ribeiro O, Sá L, Santos P, Couto L, Costa-Pereira A, Hespanhol AP. A population -based nationwide cross-sectional study on preventive health services utilization in Portugal--what services (and frequencies) are deemed necessary by patients? PLoS One 2013; 8(11):e81256.

14. Sá L, Ribeiro O, Azevedo LF, Couto L, Costa-Pereira A, Hespanhol A, Santos P, Martins C. Patients' estimations of the importance of preventive health services: a nationwide, population-based cross-sectional study in Portugal. BMJ Open 2016; 6(10):e011755.

15. Choosing Wisely Portugal [Internet]. [acessado 2020 jan 6]. Disponível em: https://www.facebook.com/ choosingwiselyportugal/. 
16. Choosing Wisely Portugal - Escolhas Criteriosas em Saúde - Ordem dos Médicos [Internet]. [acessado 2020 jan 6]. Disponível em: https://ordemdosmedicos.pt/choosing-wisely-portugal-escolhas-criteriosas -em-saude/.

17. Morgado M, Alves M, Carvalho CR, Dias CV, Sousa DC, Ferreira-Dos-Santos G, Leal I, Valente Jorge J, Vieira MB, Fortunato P, Baptista RB. Vaz-Carneiro A. Choosing Wisely Portugal: The View of Portuguese Doctors. Acta Med Port 2019; 32(7-8):559-560.

18. Portugal. Regulamento $\mathrm{n}^{\circ} 724$, de 17 de stembro de 2019. Regulamento dos Tempos Padrão das Consultas Médicas. Diário da República Eletrónico [Internet]. [acessado 2019 nov 3]. Disponível em: https://dre. pt/home/-/dre/124750410/details/3/maximized?serie $=$ II\&dreId $=124721053$.

Artigo apresentado em 04/11/2019

Aprovado em 25/08/2020

Versão final apresentada em 27/08/2020

Editores-chefes: Romeu Gomes, Antônio Augusto Moura da Silva 